\title{
The appearance of the characteristic features of mathematical thinking in the thinking of a chess player
}

\author{
Anita Misetáné Burján
}

Abstract. It is more and more important in 21st century's education that not only facts and subject knowledge should be taught but also the ways and methods of thinking should be learnt by students. Thinking is a human specificity which is significant both in mathematics and chess. The exercises aimed at beginner chess players are appropriate to demonstrate to students the mathematical thinking of 12-14 year-old students.

Playing chess is an abstract activity. During the game we use abstract concepts (e.g. sacrifice, stalemate). When solving a chess problem we use logical quantifiers frequently (e.g. in the case of any move of white, black has a move that...). Among the endgames we find many examples (e.g. exceptional draw options) that state impossibility. Affirmation of existence is frequent in a mate position with many moves. We know there is a mate but the question in these cases is how it can be delivered.

We present the chess problem on beginners' level although these exercises appear in the game of advanced players and chess masters too, in a more complex form. We chose the mathematical tasks from arithmetic, number theory, geometry and the topic of equations. Students encounter these in classes, admission exams and student circles. Revealing the common features of mathematical and chess thinking shows how we can help the development of students' mathematical skills with the education of chess.

Key words and phrases: chess thinking, mathematical thinking, abstraction, logical quantifiers, affirmation and statement of existence, talent management.

ZDM Subject Classification: A20, M10, R40.

It is more and more important in 21st century's education that not only facts of each subject, but also the ways and methods of thinking should be learnt by students. 
Obviously, in different subjects different concepts appear, therefore the ways of thinking need certainly alter slightly. Because of these differences we use the name of the subjects when talking about the form of thinking, for example we say mathematical thinking. Mathematical thinking and its didactical use has abundant literature. The most important types of mathematical thinking like the one that is generally featured, concentrating on problem solving [8], the one used in primary school education [3], the one used in secondary school education [1]. From these we tried to collect those features of mathematical thinking which are typical of mathematics only.

We chose two features which can be found in any types of thinking in a small amount but they appear in mathematical thinking to a great extent. These are the continuous use of abstraction and the use of logical quantifiers. Abstraction and logical quantifiers appear in other types of thinking too, but in mathematics in a higher rate than in any other type of thinking.

Further features of mathematical thinking can be found in book [4] and [6] which are mainly characterising mathematical thinking and related to the higher didactics of mathematics and obviously, they should be used in mathematical talent management. These are the types of stating (proving) impossibility and proving existence in cases when we do not give the existing object.

Therefore we shall below concentrate on the following features of mathematical thinking:

- The continuous use of abstraction

- The high number of logical quantifiers

- Stating (proving) impossibility

- Proving existence.

This article aims to demonstrate and prove through examples that these typical features of mathematical thinking unavoidably appear in a simple form in the youngest (12-14 year-old) students' mathematical education too. Consequently these features are present not only in secondary school or higher education but they appear already when the development of thinking enables the understanding of demonstration.

This didactical recognition is supported from another significant point of view, that is, these typical features of mathematical thinking appear and can be recognised unavoidably and in simple forms in the chess-education (chess as a subject) that gains more and more attention recently. The need and capability 
of demonstration of students under 12 are not yet developed enough for teaching them these thinking procedures within the given school facilities.

It is worth discussing the role and position of chess in a few more sentences. When examining the didactics of mathematics, the connection of chess and mathematics is not surprising because chess can be seen as part of mathematics; chess is a finite, discrete, two-player deterministic game with complete information [7].

Consequently the chess player should solve a well-defined mathematical problem for every single correct move. In simple situations - which are discussed here these can be done by a beginner chess player but in most cases even a world champion cannot solve them. Therefore players use not mathematical but other kinds of thinking in the majority of the cases when choosing their move [5]. Spreading chess as a subject is supported by the same argument that the thinking of chess players is multilayered and its different forms vary widely, therefore chess knowledge could be useful in many other thinking styles.

It is worth mentioning that the potential of using chess thinking can be recognised without any deep investigation. Forty years ago a professor of the medical university who played chess on the level of national champion emphasized that every medical student should learn to play chess, because a practicing doctor thinks as a chess player very often; even in a case of lacking information he has to make quick decisions in the procedure of healing (e.g. diagnosis or operation). Then a few years later we could read in chess journals about a chess computer programme which uses the very same principles as a medical diagnostic computer programme.

To demonstrate the appearance of the typical features of mathematical thinking we exhibit a couple of mathematical and a few chess examples. The mathematical examples are taken from their primary school curriculum of the mentioned age group, and the chess examples are taken from the curriculum of beginner chess players ([2], [9]). These examples are typical, we could find many more similar ones. The solutions are simple, therefore we highlight only the thinking methodologies used in the procedure of thinking while solving the tasks. The keywords are shown in italics. Because most reader of this article are likely to be familiar with primary school mathematics and might know less about chess, less mathematical and more chess examples are shown in the demonstrations of topics.

In chess those examples were chosen which are connected to position evaluation and which state the existence of the strategy of winning or draw. Naturally, 
in these we use the usual terminology of chess like white wins in the given position. To prove the existence of the required strategy, we discuss it on the level of beginner chess players but it is obvious that it could be discussed on the level of mathematical demonstration.

The continuous use of abstraction is very significant from the viewpoint of mathematical thinking because in mathematics, thinking is always connected with abstract concepts. In the methodology of mathematical thinking abstraction appears when a new abstract concept is created for the task.

Chess and the chess player's whole way of thinking require abstraction. Even the pieces are abstract concepts because only those features can be used which are described in their definition and these are completely independent of their shapes and names. Obviously we use actual pieces in a game but it is still said that abstraction is used because - as it was emphasized before - the chess pieces can use only those features which are included in their definition. This is supported by the fact that in different countries chess pieces have different shapes and names (Königin - queen).

The recognition of the role of abstraction in a chess player's thinking is possible in the puzzles and problems used in teaching chess.

MATHEMATICAL TASK. In a coffee roasting factory two types of coffees are roasted, one is $2500 \mathrm{HUF}$ and the other is $3300 \mathrm{HUF}$ per $\mathrm{kg}$. A $80 \mathrm{~kg}$ coffee mixture was ordered from this plant. How many $\mathrm{kg}$ has to be mixed from each type to get a mixture costing 3000 HUF? (Mathematics exercise sheet for grade 8, 19/1/2013, 11 a.m.)

The defined abstract concept in the task was the amount of one of the coffees: noted by $x$. The number $x$ can be obtained from the equation

$$
2500 x+3300(80-x)=3000 \cdot 80 .
$$

\section{Chess problem}

\section{Position:}

White: Kg1, Qe3, Re1, Ba4, a3, b2, f2, g3, h2. Black: Kf8, Qc7, Rb8, Re8, a7, b6, f7, g7, h6. White starts and wins.

Solution: 1. Qxe8+, Rxe8 2. Rxe8 matt.

In the solution the defined abstract concept during thinking is the sacrifice.

We get to a mate position with the help of this.

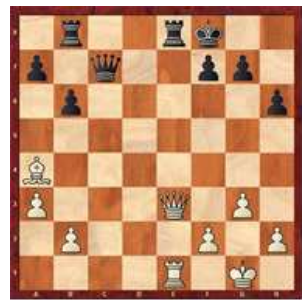




\section{Chess problem}

\section{Position:}

White: Ka1, Rd1, a4, b5, g4, h5.

Black: Ka8, Qb3, a5, b6, b7, g5, h6.

White starts and achieves draw.

Solution: 1. Rd8+, Ka7 2. Ra8+, Kxa8 stalemate.

In this position white is in significant disadvantage. In this case the realistic goal is not winning but aiming for a draw. The abstract concepts in the solution

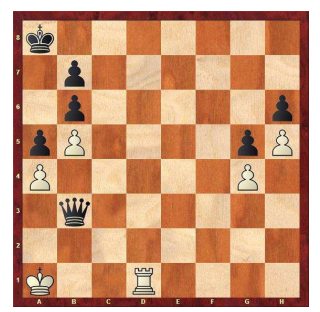
are the sacrifice (rook sacrifice in the present example) and stalemate.

In the above two positions there are no alternatives therefore they can be taught to beginner chess players, too.

Logical quantifiers (universal quantifier and existential quantifier) appear in science and in the teaching of the theory of every subject but they appear significantly more often in mathematics and in mathematical thinking.

Logical quantifiers are necessary in twosome games, for example, to define the theory of strategy (the winning or draw strategy in chess). As when someone has a strategy, he or she has a move after any of the opponent's moves and he or she will reach his or her goal with that sequence of moves. It is worth comparing this theory with the theory of the chess position where the position is not evaluated in the usual way but the player whose thinking time expires is acclaimed as loser by the competition referee. This happens only at a position where a winning sequence of moves exists, which would result in mate. When stating impossibility we will show examples for both cases.

In primary and secondary school mathematics twosome games appear only in classes with specific curriculum or after - student circles and competitions, therefore we do not present a mathematic problem from the topic of twosome games but we consider an exercise which is connected more closely to the curriculum.

Mathematical task. Prove that among any seven positive integers there are always two, whose sum or difference is divisible by 11 .

In the solution we examine the remainders of the seven numbers divided by eleven. If there are two equal remainders, the statement is proved. If any two remainders differ then there exist two of them which add up to eleven which brings us to the solution, too. 
Chess problem

Position:

White Kg2, b5. Black: Kf4. White starts and wins.

Solution: (square rule) 1. b6, Ke5 2. b7, Kd6 3. b8Q and white wins.

To reach the solution the abstract concept of distance from geometry is necessary. In the starting position the side of the base square is four units. After the move $\mathrm{b} 6$ of white the side of the changing square

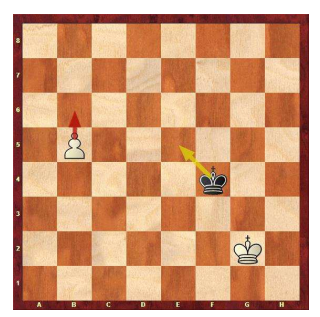
of the pawn is only three units. In the case of any black move (in the solution the best move appears which can take the black king closest to the new changing square) there exists a move for white which reduces the side of the changing square therefore the black king cannot stop the promotion of the white pawn.

\section{Chess problem}

\section{Position:}

White: Kc6, b6. Black: Kb8. White starts and wins.

Solution: 1. b7, Ka7 2. Kc7, Ka6 3. b8Q and white wins.

After the move b7 of white for any move of black there exists a winning move for white. Therefore there exists a winning strategy for white.

Comment: If we move the black king to square

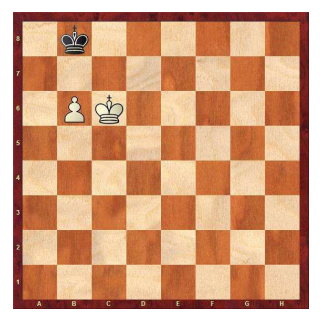
$\mathrm{c} 8$, then there is no winning strategy (as black has a draw strategy). In this case after any move of white there exists a move for black where he can keep draw. For example 1. b7, Kb8 2. Kb6 stalemate or 1. b7, Kb8 2. Kd6, Kxb7 theoretical draw or after 1. Kc5, Kb7 2. Kb5, Kb8 3. Kc6, Kc8 we reach the starting position.

Stating (or proving) impossibility is so significant in mathematical thinking that we chose a quotation to emphasize it (see [6] page 9 taken from [4]):

"When we prove that something is impossible or a problem is unsolvable, or a certain mathematical object or procedure does not exist then our thinking turns in a direction towards abstraction which reflects mathematics the most". 
Examples representing this are like cube duplication, angle trisection, and the problem of squaring the circle which were problems posed by the Greek mathematicians more than 2500 years ago, still their verification was achieved less than 250 years ago. The reason for failure is not because our capabilities or possibilities are at the time restricted but the reason is that no one ever under any kind of circumstances will be capable to solve these.

Mathematical task. Prove that $\frac{1}{18}$ cannot be expressed as a finite decimal.

When solving the exercise we use the fact that a finite decimal equals an ordinary fraction which has a denominator that is a nonnegative integer power of 10 .

$\frac{1}{18}$ is not equal with such an ordinary fraction therefore it is impossible to express it as a finite decimal.

MATHEMATICAL TASK. If we cut out two opposite squares on one of the main diagonals of a chess board then the rest cannot be covered with dominoes that cover exactly two adjacent squares.

When solving the exercise we use the fact that dominoes placed on a chess board always cover a white and a black square. As the opposite squares on the main diagonal are of the same colour there are 32 white and 30 black squares or 32 black and 30 white squares to be covered which is impossible.

These two exercises illustrate that their solution is perfectly understood by higher level students (from secondary school up) while this is not the case with 12-year-old ones. It happened that after solving the second exercise one of the brightest members of the school group tried to accomplish the task drawing it on paper after the lesson. When warning him that we had proven that the task is impossible and it was not worth trying, his answer was that he still wants to try as it might still be possible.

Within the practice of tournament referees there are situations where in a certain position one of the players requires a draw. In this case the referee has three options: rejects the draw, orders to "continue the game" postponing the decision, or accepts the draw. If we observe the next chess position from this point of view, the decision has to be postponed. Consequently the player has to demonstrate that he knows how to get to a draw position. 
Chess problem

Position:

White: Kg6, Nf6, Ne5. Black: Kg8.

Black starts and gains draw therefore white cannot find a winning strategy if black plays well.

Solution: black has two options. In the case of Kf8 it gains draw.

Note: After 1. Kh8, 2. Nf7 it is mate, so there exists a series of moves that makes black lose. If black exceeds

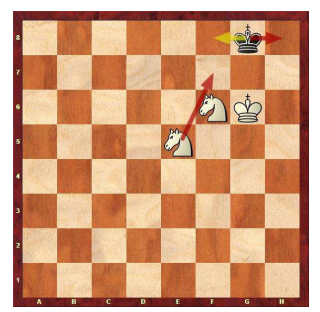
thinking time in this position then loses. After the move Kf8 if black can prove he knows the draw strategy then draw should be given.

\section{Chess problem}

\section{Position:}

White: Ka6, Bb3. Black: Ka8.

It should be proven that no matter which player starts in this position white cannot give mate, thus there is no existing sequence of moves from this position where black can be in a mate position.

Solution: If a bishop gives check then the white king has to control at least two black fields next to the

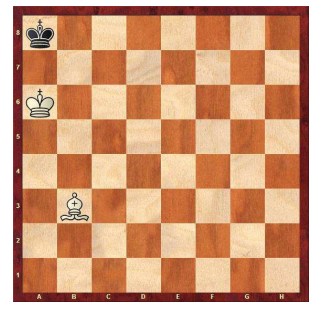
black king (here a7 and b8) which is impossible. In the case of requesting a draw the referee has to decide on a draw because there is no regular sequence of moves resulting in mate.

\section{Chess problem}

\section{Position:}

White: Kb5, Bc5, a5. Black: Kb7.

White starts and black achieves draw.

Solution: 1. a6+, Ka8 2. Kb6, Kb8 3. a7+, Ka8! White 4. Ka6, Kc6 or after Kc7 it is stalemate. If 4. Kb5 follows then the black king always remains on fields a8-b7 or stalemate follows (side pawn and wrong bishop).

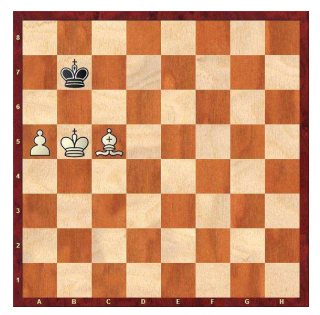

Comment: it is obvious that there is a sequence of moves from this position which results in black getting mate. If black exceeds 
thinking time in the position then loses. If he follows the above mentioned steps then proves that he knows the draw strategy and he has to be given draw.

Existence proofs have two types. One of them gives or constructs the certain object (constructive demonstration) and thus it proves existence. The second one proves the existence of the object but it does not give or construct the object itself. The second type is deeper and more abstract and typical of mathematical thinking and is called pure existence demonstration.

Miklós Laczkovich represents in the "divider game" the existence of winning strategy by a beautiful example of pure existence demonstration (in [5] page 47) but it is advised to be discussed only at higher level education.

Mathematical task. Prove that between any two distinct real numbers there is always a rational number.

The task can be solved by the next constructive proof. Obviously, without loss of generality we may assume that the given numbers are non-negative, and that if one of them is rational, then it is given as a finite decimal fraction.

Take the first digit $d$ of the larger number $A$ which differs from the digit in the same position of the smaller number $B$. Define $x$ to be the number obtained from $A$ by replacing its digits after the digit $d$ by 0 . Further, let $y$ be the number obtained from $x$ by replacing $d$ by $d-1$, and changing the rest of the digits to 9 , until the new number is already larger than $B$. Then $\frac{x+y}{2}$ is rational and it is between $A$ and $B$.

The exercise can also be solved by a pure existence proof as it was stated above: let the two numbers be $u$ and $v$ where $v>u$. Consider difference $r=v-u$. Let $q$ be a rational number smaller than $r$. In this case there is an integer $n$ such that $u<n \cdot q<v$ because if $m$ is an integer such that $m \cdot q<u$ and $v<(m+1) \cdot q$ then because of $r=v-u>q$ this would be a contradiction.

Chess problem

Position: White: Kf5, Rd5, Bf6, h6, h5.

Black: Ke8, Rh8, g5. White starts and gives mate in two moves.

Solution: In this position two cases are possible. If black has already moved the king or the rook, then 1 . Ke6 and after any move of black 2. Rd8 mate. When neither the king nor the rook have moved during the game then the last move of black could only be g5 as the pawn on 66 would have attacked the white king.

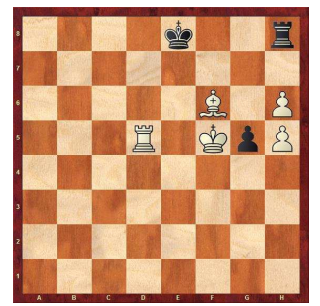


Then 1.h5xg6 (en passant) and if black castles then $2 . h 7$ mate and after any other move of black the above mentioned $\operatorname{Rd} 8$ mate. It can be seen that there is mate in two moves but we do not know how. In other words, this is an example of an existence demonstration frequently occurring in mathematics. This problem was discussed by W. Langstaff in the paper called Chess Amateur in 1922.

\section{Chess problem}

Position: White: Ka1, f5, g5, h5. Black: Ka3, f7, g7, h7.

White starts and wins.

Solution: in this position white has advance in space and both the attacking and defending kings are far from the pawns. The question is how we could break through with the pawns. 1. g6

- in the case of black not taking pawn g, after gxf7 or gxh7 the promotion of the pawn cannot be prevented

- in the case of fxg6 with 2. h6 white diverts black's g7 pawn and f pawn is freed and it is followed by the promotion of the $\mathrm{f}$ pawn.

- in the case of hxg6 the pattern is the same after 2 . f6.

\section{Conclusions}

In this paper every important part of the curriculum for 12-14 year-olds (arithmetics, number theory, geometry, equations) was included in the examples. This highlights even more the presence of mathematical thinking in this age group. Because of the same reason it is worth mentioning that there is no premathematical theory in the examples hence in the simple and important parts of the curriculum this thinking method is present. Problems in chess mathematics were avoided deliberately (only one example contains a chess board without chess pieces) though the bibliography includes a book containing problems like these ([10]). The exploration of the common elements of chess thinking and mathematical thinking helps in developing students' mathematical thinking by teaching chess.

\section{Acknowledgements}

The author is grateful to the referees for their useful and helpful remarks. 


\section{References}

[1] A. Ambrus, Bevezetés a matematika didaktikába, Egyetemi jegyzet, ELTE Eötvös Kiadó, Budapest, 2004.

[2] L. Asztalos and J. Bán, A sakkjáték elemei, Kossuth Kiadó, Budapest, 2001.

[3] I. Czeglédy, Matematika tantárgypedagógia I-II, főiskolai jegyzet, Bessenyei Kiadó, Nyíregyháza, 2000.

[4] M. Kac and S. M. Ulam, Mathematics and Logic, The New American Library, 1969.

[5] A. Kotov, Think like a Grandmaster, London, Batsford, 1971.

[6] M. Laczkovich, Sejtés és bizonyítás, TYPOTEX, Budapest, 2010.

[7] K. Varga Pásztorné and M. Várterész, A matematikai logika alkalmazásszemléletü tárgyalása, PANEM KFT, 2003.

[8] G. Pólya, A gondolkodás iskolája, Gondolat Kiadó, Budapest, 1977.

[9] L. Portisch and B. Sárközy, 600 végjáték, Sport Kiadó, Budapest, 1976.

[10] L. Sümegi, Sakk és matematika, Calibra Kiadó, Budapest, 1994.

ANITA MISETÁNÉ BURJÁN

BÁTHORI U. 1.

BALATONLELLE

HUNGARY

E-mail: anitachess64@gmail.com

(Received January, 2014) 